\title{
A New Demodulation and Modulation Method Designed for FMCW Radar
}

\author{
Wei Shen ${ }^{1,2}$ and Biyang Wen ${ }^{1}$ \\ ${ }^{1}$ School of Electronic Information, Wuhan University, Wuhan 430079, China \\ ${ }^{2}$ Department of Geosciences, University of Hamburg, Hamburg 20146, Germany
}

Correspondence should be addressed to Biyang Wen, bywen@whu.edu.cn

Received 6 December 2009; Accepted 26 January 2010

Academic Editor: John N. Sahalos

Copyright (C) 2010 W. Shen and B. Wen. This is an open access article distributed under the Creative Commons Attribution License, which permits unrestricted use, distribution, and reproduction in any medium, provided the original work is properly cited.

\begin{abstract}
An efficient demodulation method designed for FMCW (Frequency-Modulated Continuous Wave) radar is presented. It is a kind of modified DFT (IDFT) algorithm; the spectrum segment of interest can be easily extracted from the original signal without calculating the whole DFT/FFT. It provides fast demodulation and extraction of desired frequency bands in our HFSWR (HighFrequency Surface Wave Radar) system. The proposed approach enhances the performances of radar system and reduces the computing complexity. The new structure could also be inversely used for signal modulation. And also arbitrary sampling rate conversion could be achieved with the combination of forward and backward structure.
\end{abstract}

\section{Introduction}

FMCW is a radar system where a known stable frequency continuous wave radio energy is modulated by a triangular modulation signal so that it varies gradually and then mixes with the signal reflected from a target object with this transmit signal to produce a beat signal. With the advent of modern electronics, the use of Digital Signal Processing is used for most detection processing. The beat signals are passed through an Analog-to-Digital converter, and digital processing is performed on the result [1]. Fast calculating of demodulation and target detecting/tracking is an important issue in radar system design. In order to get higher Signal-to-Noise Ratio, high-speed sampling is used during Analog-Digital Converting. However, high sampling rate brings large bound of data for processing, which reduces performance of the real-time processing. SRC (Sampling Rate Conversion) method is widely used to isolate the desired channel (in frequency domain) in the communication systems [2]. Many different Digital DownConverter (DDC) structures have been implemented, and the programmable part of decimation structure includes FIR filter, comb filter, and so on. The SRC turns out to be mainly a problem of designing appropriate filters and structures implement [3]. In this paper, combining with the principle of FMCW radar demodulation and the receiver structure, an efficient demodulation structure is proposed, and further extensions are also presented.

\section{Signal Processing in FMCW Radar System}

Frequency-Modulated Continuous Wave mechanism has been widely used in modern radar system. The waveform parameter of the mechanism is described as follows:

$$
x_{T}(t)=e^{j\left(2 \pi f_{c} t+\pi \alpha t^{2}\right)}, \quad-\frac{T_{s}}{2}<t<\frac{T_{s}}{2},
$$

where $f_{c}$ is radar carrier frequency, $\alpha$ is the sweep rate, and $T_{s}$ is the sweep repetition period; the received signal is a delayed (time $t_{d}$ ) and scaled (factor $A$ ) replica of the transmit signal:

$$
x_{R}(t)=A e^{\left.j\left(2 \pi f_{c}\left(t-t_{d}\right)\right)+\pi \alpha\left(t-t_{d}\right)^{2}\right)} .
$$

The time delay $t_{d}$ can be extracted from $x_{T}(t)$ and $x_{R}(t)$, by the demodulation $x(t)=x_{R}(t) \otimes x_{T}(t)$, which is a simple 


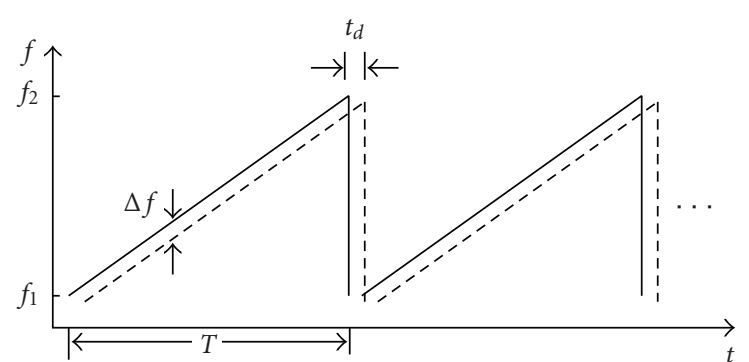

FIGURE 1: Sketch of transmit and receive signal of FMCW.

function of the target range and velocity $[4,5]$ :

$$
t_{d}(p, t, v)=F\{x(t)\}=\frac{2 R(p, t, v)}{c}=\frac{2}{c}\left[R_{o}+v\left(p T_{r}+t\right)\right],
$$

where $R_{o}$ is the initial target range, $v$ is the velocity, $c$ is the speed of the light, and $p$ is the sweep number. Figure 1 gives the sketch of transmit and receive signal of FMCW system.

As shown by Barrick, and others $[4,6,7]$, the frequency of the demodulated signal $x(t)$ is approximately proportional to target's delay time $\tau_{o}$ by

$$
\Delta f=\alpha \tau_{o} .
$$

The range of specific targets can be extracted from the frequency of demodulated signal $x(t)$. ADC is used to generate the discrete sequence $x[n]$; and according to sampling theory, in normal situation, if sampling rate $f_{s}$ is much higher than the band of desired signal $\left(0 \sim \Delta f_{\max }\right)$, the higher Signal-to-Noise Ratio can be achieved [2]. In HF radar measurement, the corresponding maximum frequency offset $\Delta f_{\max }$ (calculated from the detecting physical limitation) is only several hundred $\mathrm{Hz}$ [4]. But the radar system operates in high-frequency band. The noise outside the receiver is dominant other than noise inside the receiver. So an appropriate sampling clock is determined according to outside noise level and the SNR we can get.

\section{Principle of the Fast Demodulation in FMCW}

As discussed above, the frequency information can be extracted from Fourier Transform (FT) of $x[n] . x[k]$ may be given by

$$
x[k]=\operatorname{DFT}\{x[n]\}=\sum_{k=0}^{N-1} x[n] e^{-j 2 \pi(k n / N)}
$$

We define $W_{N}=e^{-j 2 \pi / N} ; N$ points are sampled in each repetition period. An evaluation of (5) using Fourier transform (FT) would result in $N$ points in frequency domain. But according to the maximum $\Delta f$ offset related to the maximum detecting range (as we have discussed above). Frequency resolution of FT result is $\delta F=1 / T_{s}$ [8], from the maximum frequency offset and frequency resolution. The desired frequency points are determined. The first $M$ points

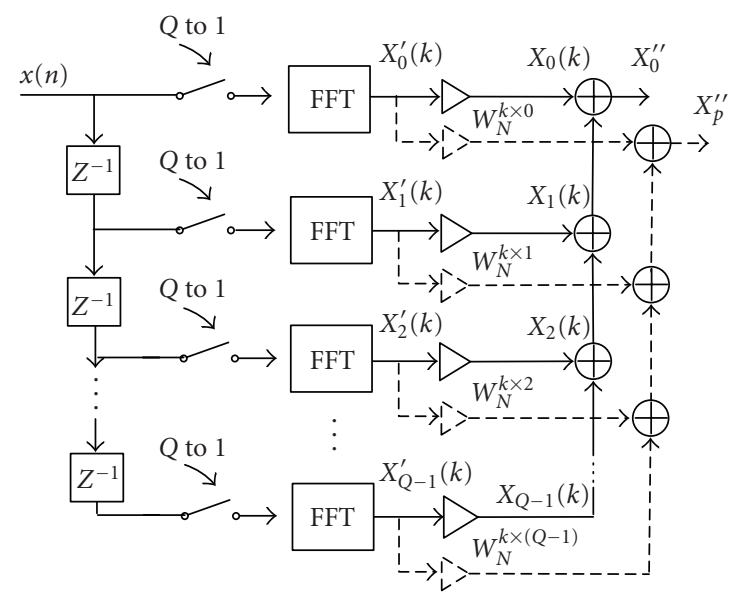

FIGURE 2: Structure of proposed demodulation method.

are enough for target detecting and further signal processing. Normally, $M$ is set to $2^{Z}$ ( $Z$ is positive integer; appropriate $M$ is selected also according to $M$-point FFT block in hardware), so we have $M$ points, and $Q=N / M, M \ll N$. If the processor is very powerful and with enough storage memory, surely, it can process the whole sequence with once FFT calculation. But usually, the system needs to process multichannel received signals and some further processing including target detecting and tracking. So the efficiency of the processing must be considered.

The new structure is described as follows: we expand

$$
\begin{aligned}
& X(k) \\
& \quad=x(0) W_{N}^{k \cdot 0}+, \ldots,+x(Q-1) W_{N}^{k \cdot Q-1} \\
& \quad+x(Q) W_{N}^{k \cdot Q}+, \ldots,+x(2 \cdot Q-1) W_{N}^{k \cdot(2 \cdot Q-1)} \\
& \quad+x[(M-1) Q] W_{N}^{k \cdot(M-1) Q}+, \ldots,+x(M Q-1) W_{N}^{k(M Q-1)} .
\end{aligned}
$$

We get the sum of each column, for example, the sum of $q$ th column $(q \in[0,1, \ldots, Q-1])$ :

$$
\begin{aligned}
X_{q} & =x(q) W_{N}^{k q}+, \ldots,+x[(M-1) \cdot Q+q] W_{N}^{[(M-1) \cdot Q+q]} \\
& =\sum_{m=0}^{M-1} x(m \cdot Q+q) W_{N}^{(k(m \cdot Q+q))} \\
& =\sum_{m=0}^{M-1} x(m \cdot Q+q) W_{N}^{k(m \cdot Q)} W_{N}^{k q} .
\end{aligned}
$$

According to the Fourier transform formula,

$$
\begin{gathered}
X_{q}^{\prime}=\operatorname{DFT}\{x[m \cdot Q+q]\}=\sum_{m=0}^{M-1} x(m \cdot Q+q) W_{M}^{k m}, \\
W_{M}^{k m}=e^{-j 2 \pi k m / M}=e^{-j 2 \pi k m /(N / Q)}=W_{N}^{k \cdot(m \cdot Q)} .
\end{gathered}
$$


From formulas (7), (8), and (9), we will get

$$
\begin{aligned}
& X_{q}(k)= X_{q}^{\prime}(k) W_{N}^{k \times q}, \\
& X_{0}^{\prime \prime}=\sum_{q=0}^{Q-1} X_{q}(k)=\sum_{q=0}^{Q-1} X_{q}^{\prime}(k) W_{N}^{k \times q}=X(k) \\
& \quad\{k=1,2, \ldots, M\} .
\end{aligned}
$$

If we separate the result of total FT of $x[n]$ into $Q$ segments, we get

$$
\begin{aligned}
X_{0}^{\prime \prime}= & X(k) \quad\{k=1,2, \ldots, M\}, \\
& \vdots \\
X_{p}^{\prime \prime}= & X(k) \quad\{k=p \times M+1, p \times M+2, \ldots,(p+1) \times M\}, \\
& \vdots \\
X_{Q-1}^{\prime \prime}= & X(k) \\
& \{k=(Q-1) \times M+1,(Q-1) \times M+2, \ldots, Q \times M\},
\end{aligned}
$$

where $p$ is a value of $(0,1,2, \ldots, Q-1)$, the Factor is defined as $W_{N}^{k \times q}(q=0,1,2, \ldots, Q-1 ; k=1,2, \ldots, M)$, and the output is $X_{0}^{\prime \prime}$. If we define

$$
\begin{gathered}
W_{N}^{k \times q} \quad(q=0,1, \ldots, Q-1 ; k=p \times M+1, \\
p \times M+2, \ldots,(p+1) \times M) .
\end{gathered}
$$

Then we can get $X_{p}^{\prime \prime}$, which is the $p$ th segment of the total FT (as shown in (10)). Figure 2 shows the block diagram of the principle of the proposed structure. $X_{0}^{\prime \prime}$ is the result of demodulation. So the $N$-point Fourier transform of sequence $x(n)$ can be replaced by the sum of a relative shorter $M$-point FFT which will be multiplied by a series of factor $W_{N}^{k \times q}$. So the analytic frequency band is converted to $F s / 2 Q$, which meets the need of the echo signal processing as analyzed in Section 2. If $p$ is defined as some certain value $(p \neq 0)$, the corresponding frequency band can be extracted from the original signal. And also if we set $p=p 1, p 2$, that is to say. we define the different factor $W_{N}^{k \times q}$ and multiplied by the result of $\mathrm{M}$-point FFT simultaneously to get multichannel output $X_{p 1}^{\prime \prime}, X_{p 2}^{\prime \prime}$, and so on. As shown in Figure 2, the dashed line means that we can add another more output channels if need.

\section{Implement in HF Radar System}

In high frequency $(3-30 \mathrm{MHz})$ ground (surface) wave radar, electromagnetic wave propagates along the ocean surface, in the lower frequency, the radar can reach a distance of 450 kilometers [9]. The propagation of surface wave is demonstrated in Figure 3.

As discussed above, appropriate sampling rate and repetition time are determined. In our HF radar system,

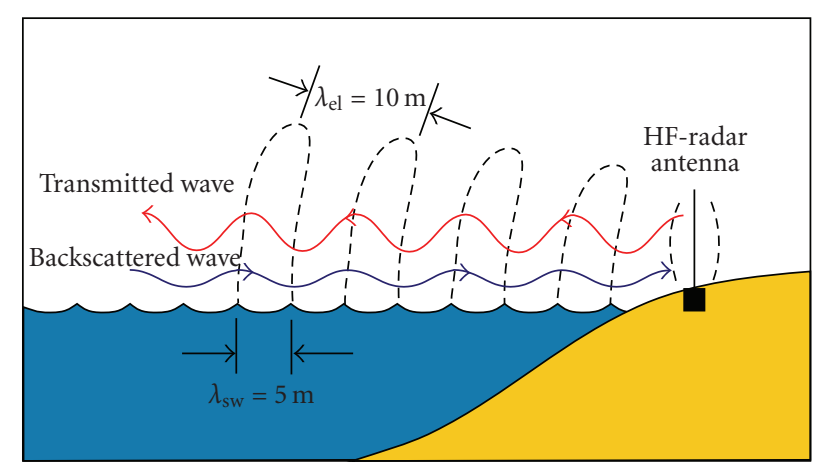

FIGURE 3: HF surface wave radar (IFM, University of Hamburg).

$N(N=8192)$ points are sampled in each sweep repetition period with sampling rate $f_{s}=60 \mathrm{KHz}\left(N=T_{s} / f_{s}\right)$. During system test, a simulated "target" is generated by delaying and attenuating transmit signal, and feed it back into the receiver. The "target" (in frequency domain) appears at the 80th point, corresponding to a distance of

$$
R_{0}=80 \times \frac{c}{2 \alpha \cdot T_{s}} .
$$

In the system, $T_{s}=0.2667 \mathrm{~s}, \alpha=112.5 \mathrm{kHz} / \mathrm{s}$, so we have a simulated target distance of $400 \mathrm{~km}$, and the offset frequency is $300 \mathrm{~Hz}\left(\Delta f=80 \times\left(1 / T_{s}\right)\right)$, but the bandwidth of sampled data is $f_{s} / 2\left(f_{s} / 2=30 \mathrm{kHz}\right)$. After processing with new structure, we get 512 points of the first frequency segment $(M=512, Q=N / M=16)$, and the analytic frequency band is decreased by $Q$ times. $f_{s}^{\prime}=f_{s} / 2 Q$, as shown in Figure $4(\mathrm{~b})$.

If there is also a target at 1000th frequency point in the spectrum, the probable distance (propagating path) is nearly $3000 \mathrm{~km}$. This might happen in Sky-wave Radar system, in which a certain range of distance is concerned. For example, Using once ionospheric reflection, an OTH (Over The Horizon) radar covers the range interval between about 1000 and $3500 \mathrm{~km}$, as shown in Figure 5. So if $p$ is set to 1 , factor $W_{N}^{k \times q}, k=[M+1, M+2, \ldots, 2 M]$. we can also get the second segment independently (Figure 4(c)). And the algorithm can be configured as multi-output structure, with different $p 1, p 2, \ldots$ value, we can get corresponding output $X_{p 1}^{\prime \prime}, X_{p 2}^{\prime \prime}, \ldots$ simultaneously.

The proposed algorithm is more efficient than the whole FFT calculation, the computing complexity of FFT is $N \log _{2}(N)$, while the complexity of proposed structure is $N \times$ $\left(1+\log _{2}(M)\right)$ for calculating $X_{0}^{\prime \prime}$. During the calculation in DSP or FPGA, FFT calculating block and parallel technique are used, so $Q$ channels $M$-point FFT can be processed simultaneously, less time is used for the processing in reality.

The conventional SRC method could also derive a certain frequency band from original signal, but the shape of digital filters (anti-imaging and anti-aliasing) is not as optimal as the proposed structure. When using conventional SRC method, with higher performance of roll-off characteristics, pass-band ripples and stop-band attenuation, higher stage number of filters is demanded [10]. However, it brings more complex computing. So the proposed method provides a 


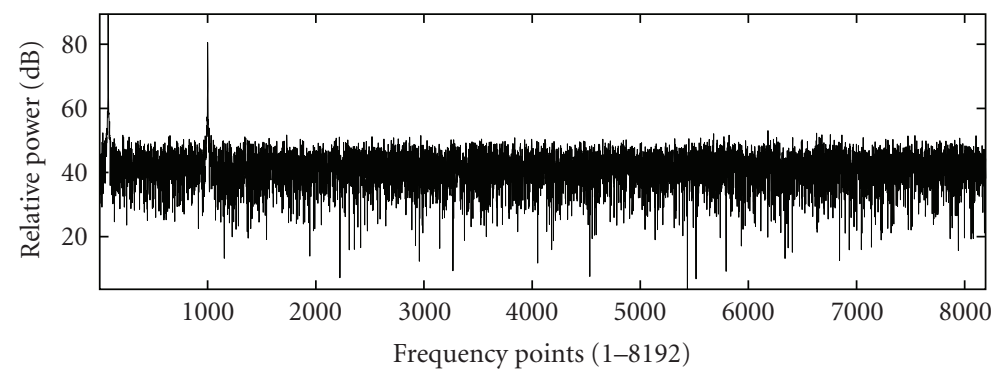

(a) Frequency points (1-8192)

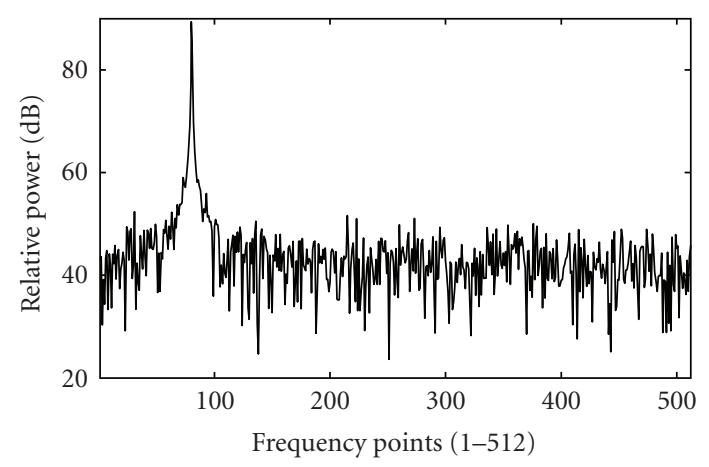

(b) Frequency points (1-512)

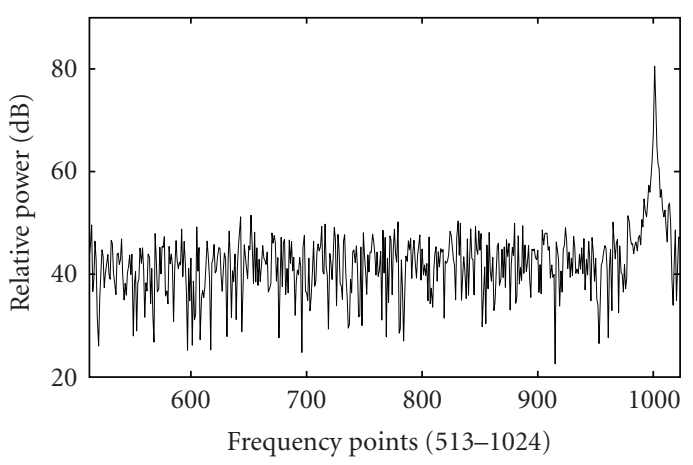

(c) Frequency points (513-1024)

FIGURE 4: Spectrum of processing results with proposed method.

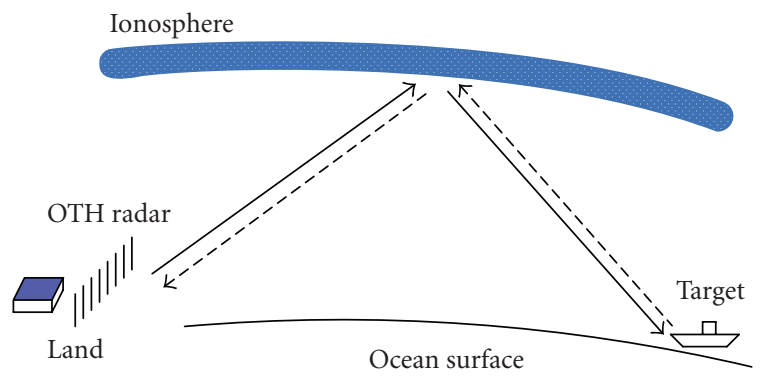

Figure 5: Demonstration of OTH Radar.

better "filter" performance (actually, it is not a kind of filter, in some case, has a function of filter) and less computation.

\section{Realization of Modulation and Arbitrary Sampling Rate Conversion}

The structure can be inversely used for modulation (interpolation), as shown in Figure 6, but the input data is $M$-point frequency domain signal $y[k]$, with the structure (Figure 6), we can get $N$-point $N=M \times Q$ time domain output signal $y[n]$, But the factor need to be changed from $W_{N}^{k \times q}$ to $W_{N}^{-k \times q}(q=0,1, \ldots, Q-1 ; k=p \times M+1, p \times M+$ $2, \ldots,(p+1) M)$, and $p$ is a value of $[0,1,2, \ldots, Q-1]$, and then we take IFFT to these $Q$ channels data to get the time domain sequences, interpolate them with $Q-1$ zeros, and shift the interpolated data by $q(q=0,1,2, \ldots, Q-1)$ respectively, at last add all the interpolated sequences, so we

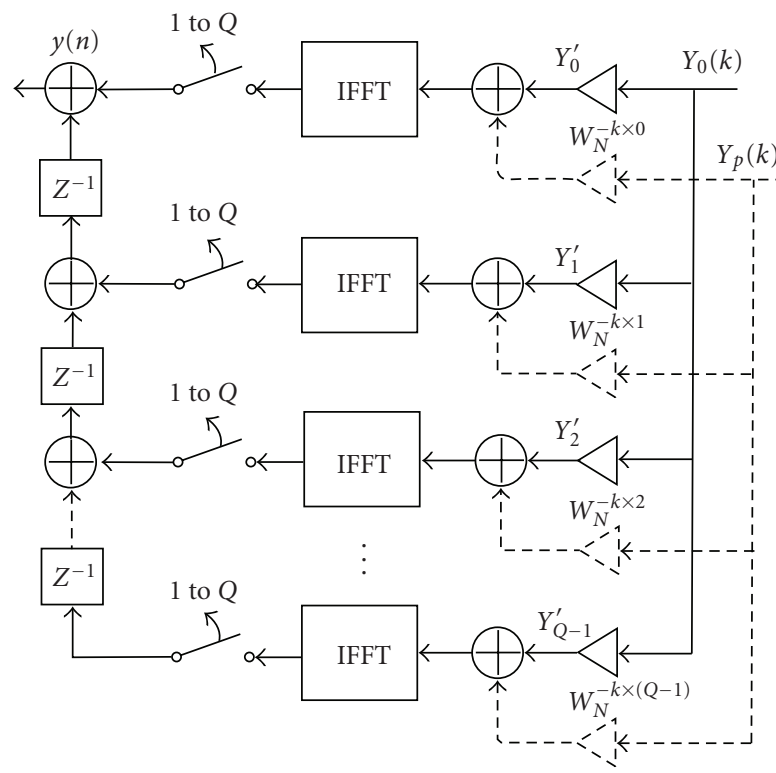

FIgURE 6: Structure of the proposed interpolation method.

can get $x_{0}[n](n=1,2, \ldots, N)$, the sampling frequency band increases by $Q$ times.

And also we can input two or even more sequences, for example, we input two sequences $Y_{1}[k], Y_{2}[k]$, and multiplied with corresponding factors $W_{N}^{-k 1 \times q}$ and $W_{N}^{-k 2 \times q}$, $(k 1=p 1 \times M+1, p 1 \times M+1, \ldots,(p 1+1) \times M ; k 2=p 2 \times$ $M+1, p 2 \times M+1, \ldots,(p 2+1) \times M)$, after the multiplication 


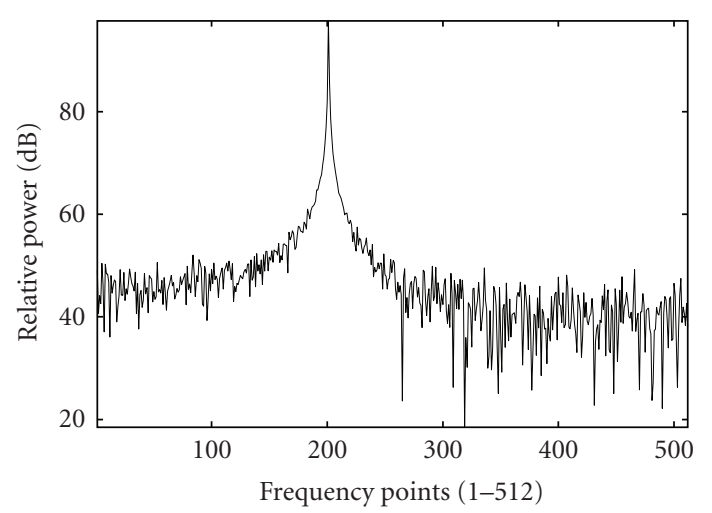

(a) Frequency points (1-512)

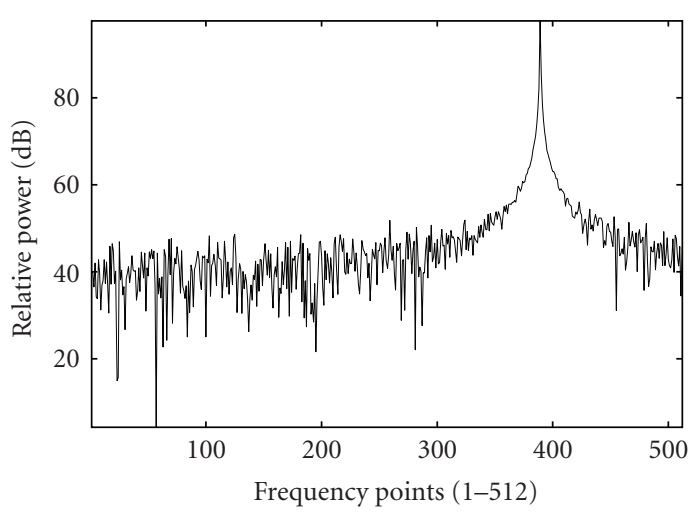

(b) Frequency points (1-512)

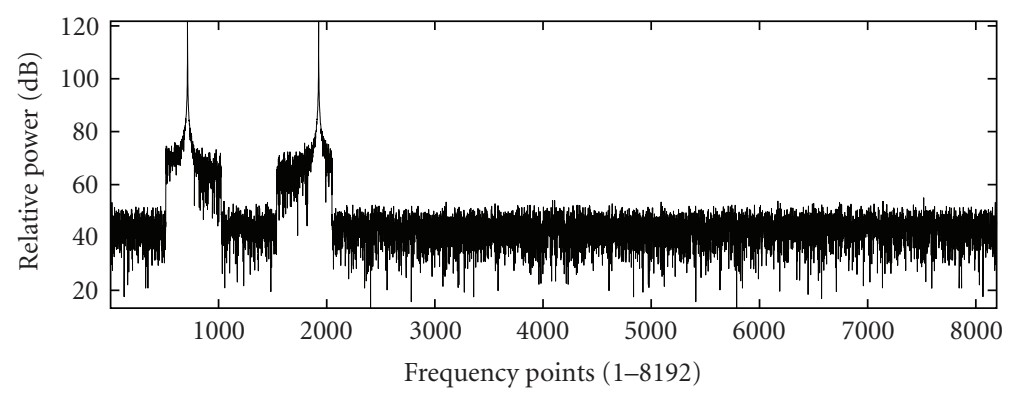

(c) Frequency points (1-8192)

FIgURE 7: Demonstration of proposed modulating interpolation.

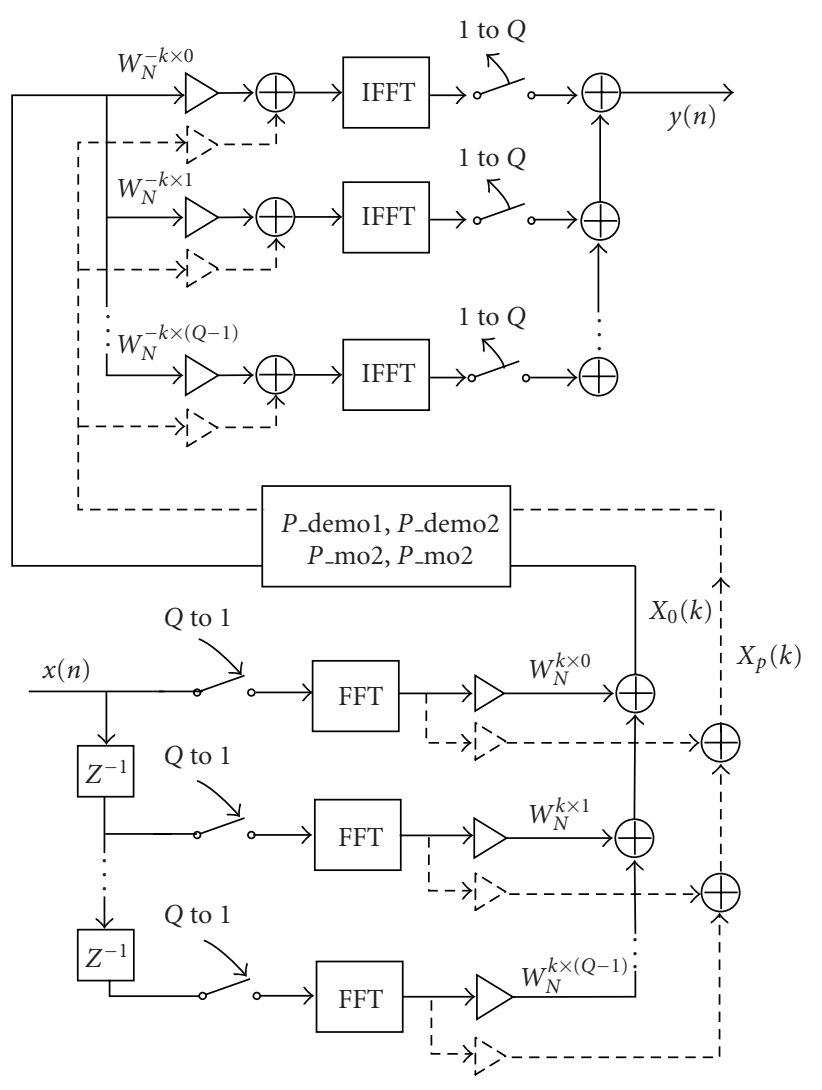

FIGURE 8: Structure of decimation and interpolation. with factors, we can add them and send into IFFT calculating block. So the two sequences are interpolated and modulated, so with the preset $p$ value, the input signal can be modulated by a certain pattern. Figure 7 demonstrates an interpolating example. Actually, the output result should be a time-domain sequence, but in order to explain the algorithm, we also give the spectrum of the output sequence. We set $p 1=1 ; p 2=3$, and after the modulation, the input signals appear in the 2nd and 4 th frequency band. The spectrum of output sequence is separated into $Q$ frequency band as shown in (10).

Combining with the decimation and interpolation methods, Arbitrary sampling rate conversion could be achieved, we define $p 1_{-}$demo $=0, Q_{-}$demo $=Q 1$ and $p 1_{-}$mo $=$ $0, Q_{-}$mo $=Q 2$ (demo is abbr. of demodulation in Figure 2, mo is abbr of modulation in Figure 6). So the total conversion rate is $Q=Q 2 / Q 1$, and also coding the frequency band unit (when certain pattern of $p 11_{-}$demo, $p 2_{-}$demo,... and $p 1_{-}$mo, $p 2_{-}$mo are predefined) could be also achieved in some communication system if it is necessary, as shown in Figure 8. All these calculations are based on the fast-calculating of $M$-point FFT (IFFT) block and parallel technique.

\section{Conclusion}

This paper describes a demodulation method to extract the desired frequency band in FMCW Radar, which is an efficient method in some periodic transceivers like radar and sonar systems. It also provides a flexible method for 
the demodulation. Different frequency bands can be derived from input signal by setting corresponding $p$ value. And we can also modulate the signal to different frequency band by inversely using the structure. All these conversion can be achieved without filters used.

\section{References}

[1] A. G. Stove, "Linear FMCW radar techniques," IEE Proceedings F, vol. 139, no. 5, pp. 343-350, 1992.

[2] S. J. Orfanidis, Introduction to Signal Processing, Prentice Hall, Upper Saddle River, NJ, USA, 1996.

[3] W. A. Abu-Al-Saud and G. L. Stüber, "Efficient sample rate conversion for software radio systems," IEEE Transactions on Signal Processing, vol. 54, no. 3, pp. 932-939, 2006.

[4] D. E. Barrick, "FM/CW radar signals and digital processing," Tech. Rep. ERL 283-WPL 26, National Oceanic and Atmospheric Administration, Boulder, Colo, USA, 1973.

[5] A. E. Carr, L. G. Cuthbert, and A. D. Olver, "Digital signal processing for target detection FMCW radar," IEE Proceedings $F$, vol. 128 , no. 5, pp. 331-336, 1981.

[6] B. J. Lipa and D. E. Barrick, "FMCW signal processing report for mirage systems," Tech. Rep., National Oceanic and Atmospheric Administration, Boulder, Colo, USA, 1980.

[7] R. H. Khan and D. K. Mitchell, "Waveform analysis for highfrequency FMICW radar," IEE Proceedings F, vol. 138, no. 5, pp. 411-419, 1991.

[8] R. de Wild, L. R. Nieuwkerk, and J. S. van Sinttruyen, "Method for partial spectrum computation," IEE Proceedings F, vol. 134, no. 7, pp. 659-666, 1987.

[9] D. W. Green, Extraction of wind speed from high frequency ground wave radar oceanic backscatter, Ph.D. dissertation, Memorial University of Newfoundland, Newfoundland, Canada, 2005.

[10] K. Walt, Mixed-Signal and DSP Design Techniques, Elsevier, Newnes, Australia, 2003. 

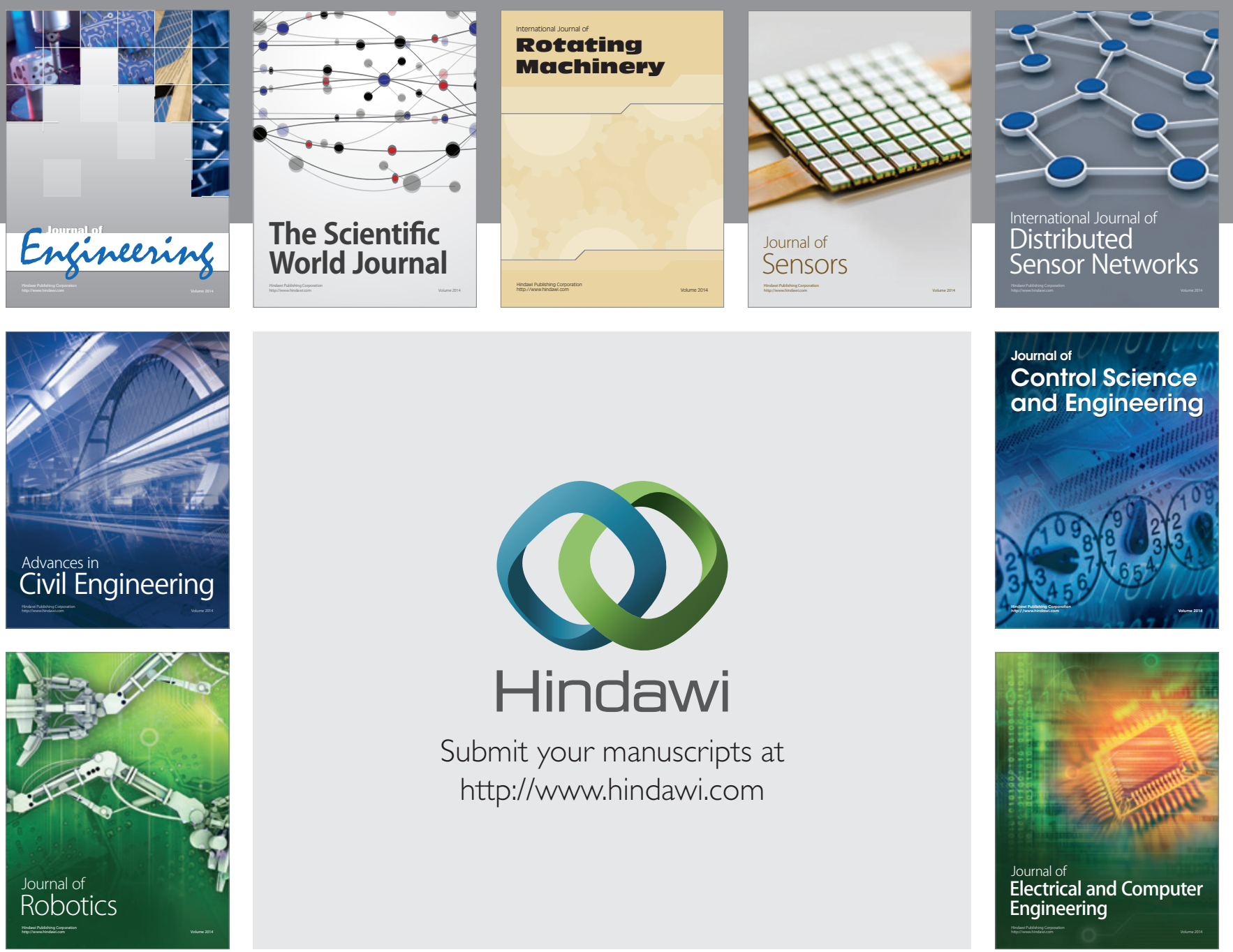

Submit your manuscripts at

http://www.hindawi.com
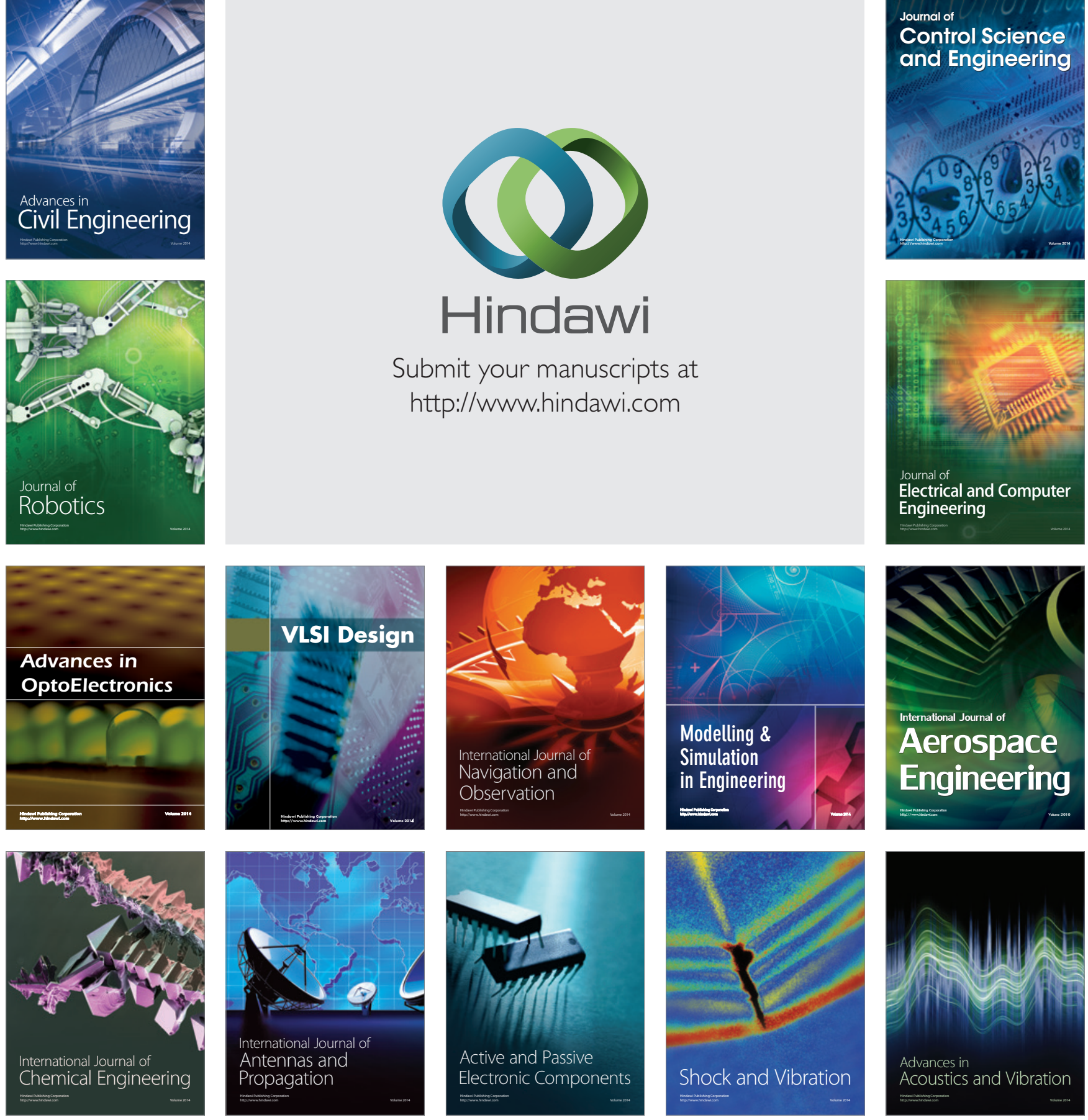